\title{
Natural Concentration in Industrial Research Collaboration
}

\author{
Bastian Westbrock ${ }^{* \dagger}$
}

January 2010

\begin{abstract}
Empirical work shows that networks of research and development alliances are asymmetric, with a small number of firms involved in the majority of partnerships. This article investigates the welfarerelevant effects of such concentrated networks in a model of network formation in an oligopolistic market. We find that concentration is a typical characteristic of a socially efficient network, when the costs of collaborative activity are significant. Moreover, expanding on prior work relating to strategically stable inter-firm networks, we compare the stable and the efficient structures. Our findings suggest that the real-world networks might even exhibit too little concentration.
\end{abstract}

\section{Introduction}

In the literature on research and development (R\&D) collaboration, there is a long tradition of work focusing on the pros and cons of an industrywide agreement as compared to independently operating research labs (e.g.,

*Utrecht University School of Economics, b.westbrock@uu.nl.

$\dagger$ This research is part of the UU High-Potential program 'Dynamics of Cooperation, Networks and Institutions' under the direction of Vincent Buskens and Stephanie Rosenkranz. I am deeply grateful to my supervisors for their financial and intellectual support. I would also like to thank the editor of the RAND Journal of Economics, Jennifer Reinganum, two anonymous referees as well as Thomas Gehrig, Jurjen Kamphorst, and Berno Büchel for their constructive comments and suggestions. This work has benefited from presentations at workshops in Aix-en-Provence, Bielefeld, Tübingen, the 2008 ZEW Conference in Mannheim, and the 2008 EEA/ESEM Congress in Milan. 
d'Aspremont and Jacquemin, 1988; Leahy and Neary, 1997). Yet, empirical work on several high-tech industries suggests that not all companies, not even direct competitors, are equally active in collaborating. Hagedoorn and Schakenraad (1992), for example, identify seven key players in the information and communication technology industries of the 1980s (AT\&T, IBM, Siemens, Philips, Fujitsu, NEC, and Olivetti), who are involved in many of the collaborative partnerships in these industries. Similarly, for the global biotech and pharmaceutical industries of the 1990s, Powell, Koput, White, and Owen-Smith (2005) find that a group of 24 well-known players had each formed more than twenty strategic alliances, whereas the majority of firms formed less than two.

These observations raise a number of questions. Can we explain the emergence of a concentrated market structure in a model of R\&D collaboration between firms? Is concentration welfare-efficient? Finally, in the light of the favorable treatment of research joint ventures in the U.S. and in Europe, is there a policy lesson to learn from such an analysis? For example, should $\mathrm{R} \& \mathrm{D}$ collaboration be encouraged between the firms on the periphery of an industry or rather between the central players?

Despite the empirical evidence, issues like these have not been sufficiently addressed in the literature. Katz (1986) was the first to study a less than industry-wide collaborative agreement. More recent contributions come from Bloch (1995) and Yi (1998), who investigate the set of industry partitions into research coalitions, and Goyal and Moraga-Gonzáles (2001) and Goyal and Joshi (2003), who study networks of bilateral R\&D alliances. A common finding is that less than industry-wide agreements are typical market structures in equilibrium. Of particular interest are the analyses presented by Goyal and Joshi (2003). The authors find highly asymmetric structures in equilibrium, where some firms are even completely excluded from any collaborative activity.

Goyal and Joshi (2003) investigate a game of network formation in a homogeneous-product oligopoly. In the first stage of the game, the firms can form bilateral collaborative links between each other, before in the second stage all firms, even the collaborators, compete in the product market. A collaborative tie leads to a reduction in marginal production costs for the firms involved. However, the firms have to incur some costs of link formation. The authors model this cost to be significant as compared to the returns from the unit-cost reduction. Yet, the cost is fixed and remains the same across all links. 
This article investigates the socially efficient networks in the model of Goyal and Joshi (2003) and shows that asymmetric networks are typically efficient as well. ${ }^{1}$ Our first result pertains to the architecture of the efficient network (Proposition 1). The efficient network may be the empty network, in which no firm collaborates, or the complete network with a collaborative agreement between any pair of firms. But if it is neither of these, the efficient network has a dominant group or an inter-linked star architecture, both of which exhibit a strong asymmetry between firms. ${ }^{2}$

Second, we examine the density and the degree variance in the efficient network. The results of this analysis show that social welfare in a network can be expressed as an additive function of the density and the degree variance in the network (Lemma 2). Whereas the density captures the welfare contribution from the number of collaborative links, the degree variance depicts the contribution from their dispersion among the firms. Moreover, as welfare is positively associated with degree variance, our analysis implies that in an efficient network a given number of collaborative ties is maximally concentrated around a subset of the firms in a market (Proposition 2). Even though the exact values of the density and the degree variance depend on the characteristics of demand and the costs of forming links, our subsequent steps show that the characterization applies to a wide range of market settings. There exists a generic set of parameters that supports an efficient network with a highly unequal dispersion of links (Proposition 3).

The intuition underlying these findings is derived from a fundamental property of the demand for cost-reducing R\&D investments. The seminal articles by Arrow (1962) and Dasgupta and Stiglitz (1980) already point to the indivisibility of research output and its implications for the market structure: an invention, for example a cost-reducing one, can be applied to every unit of a firm's product, regardless of its scale of production. The social returns from an R\&D investment are therefore higher, the larger the firm's scales. This motivates some properties of tie formation in a network between oligopolistic firms. First, the social returns of creating a collaborative tie between two firms are higher, the larger the firms' market shares. Second, the reallocation of ties from a firm at the fringe of the market to a dominant

\footnotetext{
${ }^{1}$ The notions of efficiency investigated in this article are the consumer surplus and the total surplus. In fact, we analyze a more general market setting than the one investigated in Goyal and Joshi (2003). Our model bases on Singh and Vives' (1984) demand for differentiated products and, therefore, considers richer types of product market interaction.

${ }^{2}$ The network structures are illustrated in Figure 1.
} 
market player is socially desirable. Furthermore, because a firm's market share improves in its stock of collaboration ties, the gradual construction of the network is subject to a self-reinforcing dynamic, which favors the formation of highly concentrated structures.

In a subsequent analysis, we compare the structure of the efficient network with the one of the strategically stable networks characterized in Goyal and Joshi (2003, 2006). The comparison shows that stable networks with a small or moderate density are typically inefficient. However, because of the complex structure of the efficient network in markets with a finite number of firms, our formal results pertain only to the limiting case of markets with a continuum of firms. In such a market, every stable network is inefficient that consists of less than half of the maximal attainable links (Propositions 4 and 5). As suggested by our previous findings, the reason for this failure in the network structure is that, even though the stable networks may be highly asymmetric, they still exhibit too little concentration.

Building on the last point, we develop some implications for the design of effective policy programs to foster R\&D collaboration between firms. The major value of this article is, however, its contribution to the existing studies on collaboration networks in oligopolistic markets, when the costs of link formation are significant. Goyal and Moraga-Gonzáles (2001) and Deroïan and Gannon (2006) provide a complete characterization of the strategically stable and the efficient structures in the set of regular networks, where all firms have an equal number of links. Moreover, Goyal and Joshi (2003, 2006) characterize the strategically stable structures among all (regular and irregular) networks and show that they are typically irregular. The current study identifies the efficient structures in the set of all networks.

Furthermore, the article is one of the few to point out the social benefits from concentrating costly joint research activities around a small number of firms in a market. ${ }^{3}$ An implication of our findings for the analyses in Bloch

\footnotetext{
${ }^{3}$ The analyses of some specific market settings in Goyal and Moraga-Gonzáles (2001) and Goyal and Joshi (2003) point in this direction as well. On the one hand, Goyal and Moraga-Gonzáles (2001) show for a Cournot-triopoly with significant costs of link formation that an asymmetric collaboration network can be efficient. Goyal and Joshi (2003), on the other hand, find for a Bertrand-oligopoly and insignificantly small linking costs that the efficient network has an inter-linked star architecture. Our analysis indicates that their findings carry over to more general market settings, where at the same time there are an arbitrary number of firms, differentiated products, and significant linking costs.
} 
(1995) and Yi (1998) is that the exclusion of some firms from collaborating can be socially desirable. The authors point to the welfare losses from an asymmetric market structure in a model, where the costs of collaboration are insignificantly small and the industry-wide agreement is efficient. In contrast, the current study investigates the situation of large costs, for which the complete network is inefficient.

Finally, there is also a link between this article and the sociological literature on social networks. One of the focuses of this literature is to propose different network measures and to assert the suitability of each of these measures to different situations. ${ }^{4}$ We provide a micro-economic foundation for the use of two simple measures in the study of inter-firm alliance networks: Lemma 2 suggests that the welfare-relevant properties of such a network are completely captured by its density and degree variance, where the last is one way to measure the centralization in a network (Snijders, 1981).

The remainder of the article is organized as follows. Section 2 introduces some network terminology, and Section 3 contains the description of the model. The efficient network is characterized in Section 4. Section 5 compares the efficient network with the structure of the strategically stable networks, and Section 6 provides some robustness checks of the main findings. Section 7 concludes.

\section{Networks}

Consider a set of initially identical firms, $N=\{1,2, \ldots, n\}$, with $n>2$. For any distinct $i, j \in N$, a pairwise relationship between the firms is depicted by a bilateral link $i j$, and a network $g=\{i j: i, j \in N, i \neq j\}$ is the complete collection of the links between firms. To denote network $g \cup\{i j\}$, which is obtained from $g$ by adding link $i j$, write $g+i j$. Likewise, the network that is obtained from $g$ by subtracting link $i j$ is depicted by $g-i j$. Furthermore, denote by $N_{i}(g)$ the set of firms, with which firm $i$ has a link in $g$; firm $i$ 's degree is defined as the cardinality of this set, $\eta_{i}(g)=\left|N_{i}(g)\right|$.

A network partitions the set of firms according to their degrees. Distinct $i, j \in N$ are members of the same group $h_{l}(g)$ of the degree partition $\left\{h_{0}(g), h_{1}(g), \ldots, h_{m}(g)\right\}$ if and only if $\eta_{i}(g)=\eta_{j}(g)=l$, where $0 \leq l \leq m$. Define the degree distribution of a network as the function that assigns to

\footnotetext{
${ }^{4}$ See Wasserman and Faust (1994) for an overview of the measures used in social network analysis.
} 
every nonnegative integer $l$ the frequency weight $n_{l}(g)=\left|h_{l}(g)\right| / n$. Two important characteristics of the degree distribution are:

- the density $D(g)=\bar{\eta}(g) /(n-1)$, where $\bar{\eta}(g)=\sum_{i \in N} \eta_{i}(g) / n$ denotes the average degree and $0 \leq D(g) \leq 1$, and

- the normalized degree variance $C(g)=V(g) / \hat{V}(n)$, where the degree variance, $V(g)=\sum_{i \in N}\left(\eta_{i}(g)-\bar{\eta}(g)\right)^{2} / n$, is normalized by its maximum given $n$ firms, $\hat{V}(n)$, such that $0 \leq C(g) \leq 1 .^{5}$

The density measures the overall connectivity between the firms in a network, whereas the (normalized) degree variance is a measure of network concentration or network centralization, i.e. it captures the extent to which some firms are more central than others in the network (Snijders, 1981).

In a regular network, $g^{r}$, of density $D$ every $i \in N$ has a degree of $\eta_{i}\left(g^{r}\right)=(n-1) D$. Hence, $g^{r}$ does not exhibit any concentration, $C\left(g^{r}\right)=0$. Otherwise, a network $g$ is called irregular if and only if $C(g)>0$. Two examples of regular networks are the empty network, $g^{e}$, where $\eta_{i}\left(g^{e}\right)=0$ for all $i \in N$, and the complete network, $g^{c}$, with $\eta_{i}\left(g^{c}\right)=n-1$.

Another characteristic of a network is its architecture. Two networks $g$ and $g^{\prime}$ share the same architecture, if there exists a permutation of firms, $P$, such that $g^{\prime}=\{P(i) P(j) \mid i j \in g\}$. Thus, network $g^{\prime}$ should be attainable from $g$ by just relabeling the firms. An example of an irregular architecture, which is important in this study, is the dominant group architecture of size $n^{\prime}$, $g^{n^{\prime}}$, with $2 \leq n^{\prime} \leq n-1$. The dominant group architecture is first introduced in Goyal and Joshi (2003) and consists of two groups of firms, $\left\{h_{0}, h_{n^{\prime}-1}\right\}$; one in which every firm is linked to every other firm in that group (denoted by $h_{n^{\prime}-1}$ ), and a second group consisting of isolated firms (denoted by $h_{0}$ ). The authors also introduce the class of inter-linked star architectures. An inter-linked star, $g^{x}$, induces a degree partition, where there are two or more groups of firms with a positive degree. The group of firms with the highest degree in $g^{x}$ is denoted the center group, $h_{c}=h_{m}\left(g^{x}\right)$. Every other firm with a positive degree belongs to the periphery, $h_{p}=\bigcup_{0<l<m} h_{l}\left(g^{x}\right)$. Moreover, each firm in the center group is linked to every firm with a link. Hence, let $i \in h_{c}$. If $\eta_{j}\left(g^{x}\right) \geq 1$, then $i j \in g^{x}$. A prominent example of an inter-linked star

\footnotetext{
${ }^{5}$ Snijders (1981) determines the maximum degree variance, which is given by $\hat{V}(n)=$ $\frac{(3 n-2)^{2}(n-2)(3 n+2)}{256 n^{2}}$ for a network with $n$ firms.
} 


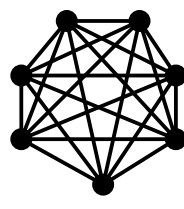

Complete $(D=1, \mathrm{C}=0)$

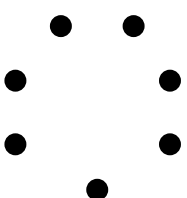

Empty $(D=0, \mathrm{C}=0)$

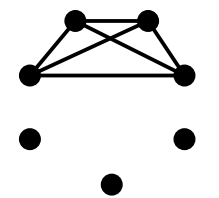

Dominant group

with n'=4

$(D=0.24, \mathrm{C}=0.66)$

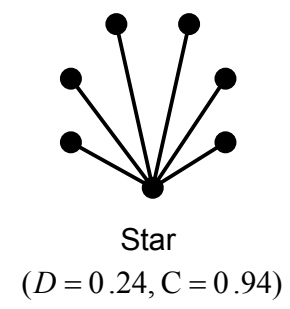

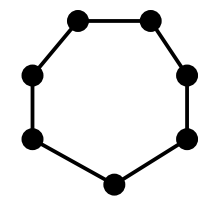

Regular ( $D=0.29, \mathrm{C}=0)$

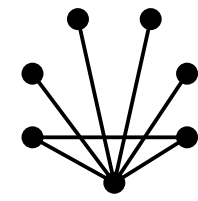

Inter-linked star with one center and two peripheral groups $(D=0.29, \mathrm{C}=0.88)$

Figure 1: Network structures

architecture is the star, $g^{s}$, which consists of two groups of firms, $\left\{h_{1}, h_{c}\right\}$, with $\left|h_{c}\right|=1$. The defined networks are illustrated in Figure 1 .

\section{The model}

In the following, we describe and investigate a generalized version of the two-stage model introduced in Goyal and Joshi (2003). In the first stage, a social planner forms a network of marginal cost-reducing links between firms, which requires the investment of a fixed cost per link. In the second stage, the firms compete in the product market. The model of the product market investigated in this article is more general than the one of Goyal and Joshi (2003). Whereas the authors investigate a homogeneous-product Cournot oligopoly, we build on the market model by Singh and Vives (1984), which allows for price or quantity competition between firms as well as for differentiated products.

We first present the model and solve its second stage. Some of the questionable assumptions underlying the model are highlighted in the text and discussed in Section 6. Let us point out already here that none of these 
are essential and can all be relaxed without eroding the main results of this study.

Market competition. In the second stage, each firm $i \in N$ sells a single, possibly differentiated, product to a continuum of homogeneous consumers, who consume each a numeraire good $I$ in addition. Let $q_{i}$ denote the quantity and $p_{i}$ the price of good $i$. A representative consumer maximizes:

$$
U\left(I, q_{1}, \ldots, q_{n}\right)=I+\alpha \sum_{i \in N} q_{i}-\frac{1}{2} \sum_{i \in N} q_{i}^{2}-\frac{\beta}{2} \sum_{i \in N} \sum_{j \neq i} q_{i} q_{j},
$$

under the constraint $I \leq-\sum_{i \in N} p_{i} q_{i}$. The parameter $\alpha$ captures the total size of the market, whereas $\beta$, a real number from the interval $(0,1]$, denotes the degree of substitutability between products. Thus, the analysis is restricted to markets where the collaborating firms are competitors in the product market. In particular, $\beta=1$ depicts a market of perfect substitutable goods, and $\beta \rightarrow 0$ represents the case of almost independent markets. From standard utility maximization, we arrive at a system of inverse demand functions $p_{i}=\alpha-q_{i}-\beta \sum_{j \neq i} q_{j}, i \in N$. A firm's profit, gross of linking costs, is given by $\pi_{i}=\left(p_{i}-c_{i}\right) q_{i}$.

Let the firms compete either in quantities or in prices, but let us disregard the case of perfect Bertrand competition, which means that under price competition we assume $\beta \in(0,1)$. In either case, the Nash equilibrium quantities can be expressed in the form $\lambda q_{i}=\mu \alpha-\nu c_{i}+\xi \sum_{j \neq i} c_{j}$. Using subscript $q$ and $p$ to denote competition in quantities and prices respectively, the parameters are given by:

$$
\begin{aligned}
\lambda_{q} & =1, \quad \mu_{q}=\frac{1}{2+(n-1) \beta}, \quad \nu_{q}=\frac{2+(n-2) \beta}{(2+(n-1) \beta)(2-\beta)} \\
\xi_{q} & =\frac{\beta}{(2+(n-1) \beta)(2-\beta)},
\end{aligned}
$$

and

$$
\begin{aligned}
\lambda_{p} & =\frac{(1-\beta)(1+(n-1) \beta)}{1+(n-2) \beta}, \quad \mu_{p}=\frac{(1-\beta)}{2+(n-3) \beta} \\
\nu_{p} & =\frac{2+(3 n-6) \beta+\left(n^{2}-5 n+5\right) \beta^{2}}{(2+(n-3) \beta)(2+(2 n-3) \beta)} \\
\xi_{p} & =\frac{(1+(n-2) \beta) \beta}{(2+(n-3) \beta)(2+(2 n-3) \beta)} .
\end{aligned}
$$


Prices and profits are in equilibrium $p_{i}=\lambda q_{i}+c_{i}$ and $\pi_{i}=\lambda q_{i}^{2}$, respectively. The following assumption ensures that, regardless of the precise network structure, no firm exits the product market:

Assumption 1. No market exit: under any attainable profile of marginal production costs, induced by a network of collaborative ties, all firms produce positive quantities in equilibrium. ${ }^{6}$

Links and cost reduction. In the first stage of the model, the social planner designs a collaboration network between firms. We interpret a link in this network as a bilateral R\&D joint venture or a cross-licensing agreement, where the purpose is the joint development or the exchange of a process technology. Hence, links are formed in order to reduce the marginal production costs of the firms.

Throughout the article, we make the simplifying assumption that this cost reduction is exogenous. Thus, a network $g$ directly induces a vector of marginal production costs, $c(g)=\left\{c_{1}(g), c_{2}(g), \ldots, c_{n}(g)\right\}$. This assumption is not uncommon in the literature on $\mathrm{R} \& \mathrm{D}$ collaboration and has been motivated by more detailed models of collaborative agreements (e.g., Bloch, 1995; Goyal and Joshi, 2003). Bloch (1995), for example, motivates exogeneity by a simple model of a joint venture between $a$ firms, $a>1$. The members of a venture can set up a joint research facility to which all of them have unrestricted access. Bloch assumes that, due to capital market imperfections, each firm can invest only a fixed amount $r$ in the facility. Moreover, the output of the facility, $z(r)$, exhibits constant returns to scale and leads to a reduction of the members' marginal production costs. Hence, in a joint venture of size $a$, the members' costs are reduced by $a z(r)$. Adapting this model to the case of bilateral agreements, it follows that each link leads to an exogenous cost reduction of $2 z(r)$. In addition, we make the following two assumptions regarding the R\&D technology. Denote the linking costs of firm $i$ in network $g$ by $F_{i}(g)$. For any network $g$ and $i j \in g$ :

Assumption 2. Constant link returns: $c_{i}(g+i j)-c_{i}(g)=c_{j}(g+i j)-c_{j}(g)=$ $-\gamma$ and $F_{i}(g+i j)-F_{i}(g)=F_{j}(g+i j)-F_{j}(g)=f / 2$, where $\gamma>0$ and $f>0$;

Assumption 3. No inadvertent spillovers: $c_{k}(g+i j)-c_{k}(g)=0$ for any $k \in N \backslash\{i, j\}$.

\footnotetext{
${ }^{6}$ The precise condition depends on the mode of competition and the productivity of collaborative ties. This condition will be specified in footnote 7 .
} 
We therefore write more conveniently $F_{i}(g)=\frac{1}{2} f \eta_{i}(g)$ and $c_{i}(g)=\gamma_{0}-$ $\gamma \eta_{i}(g)$, where $\gamma_{0}>(n-1) \gamma$. Both assumptions will be relaxed in Section 6 .

Efficiency. It follows from the preceding that given a network $g$ the net profits and consumer utility can be expressed as functions of the firms' degrees in $g:^{7}$

$$
\Pi_{i}(g)=\pi_{i}\left[\eta_{i}(g), \sum_{j \neq i} \eta_{j}(g)\right]-\frac{1}{2} f \eta_{i}(g) \quad \text { and } \quad U(g)=U\left[\eta_{1}(g), \ldots, \eta_{n}(g)\right] .
$$

Networks are investigated in terms of both social welfare concepts, the consumer surplus (net of linking costs) and the total surplus. A network $g^{*}$ is therefore defined efficient, if respectively $U\left(g^{*}\right)-\frac{1}{2} f \sum_{i \in N} \eta_{i}\left(g^{*}\right) \geq U(g)-$ $\frac{1}{2} f \sum_{i \in N} \eta_{i}(g)$ or $U\left(g^{*}\right)+\sum_{i \in N} \Pi_{i}\left(g^{*}\right) \geq U(g)+\sum_{i \in N} \Pi_{i}(g)$ for all $g$. For expositional simplicity, we denote the total surplus and the consumer surplus in a network by $W(g)$ throughout the following sections, unless a distinction is necessary.

\section{The efficient network}

In this section, we characterize the efficient network structure of the model. The difficulty with the analysis is that, even though we disregard knowledge spillovers, the marginal social returns of any two collaborative links are interrelated by the fact that the collaborating firms are, also at the same time, competitors in the product market.

We show that this market-based interdependence between links has two faces: the social welfare function is convex as well as submodular with respect to collaborative links. We apply these properties to characterize the architecture of the efficient network. Subsequently, we express social welfare in a network as a function of two characteristics of its degree distribution, the density and the degree variance. The specific form of this functional dependence allows us to derive the important result that a concentration of collaborative ties is a typical feature of the efficient network. We conclude the section with an analysis of the dependence of the efficient network on the precise cost and demand parameters.

\footnotetext{
${ }^{7}$ In order to satisfy Assumption 1, we need to assume that in the dominant group architecture $g^{n-1}$, where only firm $i$ is isolated, $q_{i}\left(g^{n-1}\right)>0$. This implies for quantity competition $\alpha-\gamma_{0}>\frac{\gamma(n-1)(n-2) \beta}{2-\beta}$, and for price competition $\alpha-\gamma_{0}>\frac{\gamma(1+(n-2) \beta)(n-1)(n-2) \beta}{2+(2 n-5) \beta-(2 n-3) \beta^{2}}$.
} 
Network architecture. In a first step, we investigate the marginal social benefits of forming collaborative links between firms, taking an arbitrary network structure as being given. For this purpose, it is helpful to think of a collaborative tie $i j$ as a cost-reducing investment by firms $i$ and $j$. The marginal social benefits of this investment increase with the scales of production at the firms, prior to that link, because the output of the collaboration, i.e. a process innovation, can be applied to every unit of their products. However, in an oligopolistic market, the quantities of firms $i$ and $j$ are determined by all of their other collaborative ties as well as the links of all their competitors, making the returns dependent on the structure of the whole network. This market-based interdependence between collaboration links is demonstrated in two properties of link formation.

Lemma 1. For any network $g$ with $i j, i k, k l \notin g$, any $i, j \in N$ and $k, l \in$ $N \backslash\{i, j\}$ :

(i) the total surplus and the consumer surplus are convex in links: if $\eta_{j}(g) \geq \eta_{k}(g)+1, W(g+i j+i k)-W(g+i k)>W(g+i k)-W(g) ;$

(ii) the total surplus (under price and quantity competition) and the consumer surplus under price competition are submodular in links: $W(g+$ $i j+k l)-W(g+k l)<W(g+i j)-W(g){ }^{8}$

The proof of the lemma can be found in the Appendix. Convexity follows from the fact that firm $i$ attains a larger market share in network $g+i k$ than in $g$. Hence, the subsequent process innovation aligned with the addition of link $i j$ affects a larger output of firm $i$. However, convexity requires that firm $i$ 's subsequent partner, firm $j$, is at least as well connected in network $g+i k$ as firm $i$ 's previous partner, firm $k$, is and therefore produces at least the same quantity. Submodularity means that the social returns to a link $i j$ deteriorate with the formation of a link between any other pair of firms. The reason is that firm $i$ 's and $j$ 's market shares are declining in the number of links of their rivals. ${ }^{9}$

\footnotetext{
${ }^{8}$ The consumer surplus is submodular under quantity competition as well, requiring $\beta<(n-2) /(n-1)$.

${ }^{9}$ Note that the central arguments behind convexity and submodularity are the constant unit-cost reductions of the collaborating firms and the resulting changes in market shares. Thus, Lemma 1 is likely to also hold in the context of a more general demand system than the linear one chosen for this study.
} 
The two properties allow for a marginal check of efficiency, which is in the same spirit as the stability check introduced in Goyal and Joshi (2003, 2006). The idea is to check whether a given network is socially desirable or whether an additional collaboration tie can increase welfare. This results in a meaningful selection among the network architectures.

Proposition 1. If the efficient network $g^{*}$ is regular, then it must be either the empty network, $g^{e}$, or the complete network, $g^{c}$. If the efficient network $g^{*}$ is irregular, it has either a dominant group architecture, $g^{n^{\prime}}$, for some $2 \leq n^{\prime} \leq n-1$, or an inter-linked star architecture, $g^{x}$.

The proof is presented in the Appendix, and the networks included in the set of efficient architectures are illustrated in Figure 1. The proof is based solely on the convexity of the social welfare function. Due to this property, a firm $i$ with at least a single link in network $g$, say to a firm $k$, should be connected to every firm $j$ with the highest degree in the network. The reason is that $\eta_{j}(g-i k) \geq \eta_{k}(g-i k)+1$, so that Lemma 1 (i) can be applied. Hence, a commonality of all efficient network architectures is that there exists a group of firms (the center group), where each firm in this group has the highest degree and is connected to every other firm with a link.

An important class of architectures that is excluded by the proposition are the regular networks, which are non-empty and incomplete. The reason is that in a regular network the center group consists of every $i \in N$. Yet, only in the complete network are the firms completely connected to each other. From the class of irregular networks, any architecture is ruled out that consists of a center group and a periphery of firms, but where either the firms in the center are not completely connected with each other or where any one firm in the periphery is not linked to all the center firms. Hence, in an efficient architecture, the central firms are densely connected through a web of direct links and, in the case of an inter-linked star, indirect links via the peripheral firms.

Two remarks about Proposition 1 are noteworthy: first, as suggested by our analysis below, the characterization of the result is tight in the sense that there is no architecture included in the set of efficient architectures that is not efficient for some parameters. Second, even though the proof of the result does not take advantage of the submodularity of the welfare function, this property crucially shapes the architecture of an efficient network. Whereas convexity renders the formation of links between the firms that are already involved in a lot of ties efficient, submodularity dilutes the benefits of also 
linking the poorly connected firms. Hence, submodularity favors the selection of highly concentrated dominant group architectures or inter-linked star architectures, where collaborative activity is concentrated around a small group of firms.

Because these architectures differ considerably in terms of the size of that group and the degrees of the firms contained in it, it is still an open question which architecture is desirable. Moreover, it is still not clear whether some cost and demand parameters exist for which a dominant group or an interlinked star architecture can actually be supported as a welfare-maximizing network. These issues are investigated below.

Degree distribution. Here, we determine the properties of the degree distribution in a socially efficient inter-firm network. As it is assumed that the marginal production costs only depend on the number of collaborative ties of a firm, it is clear that any two networks with the same degree distribution also produce the same total surplus. The analysis of the relationship between the degree distribution and social welfare can, therefore, provide further insights into the structure of an efficient network.

Generally, the welfare in any network $g$ can be decomposed into two additive terms:

$$
W(g)-W\left(g^{e}\right)=\left[W\left(g^{r}\right)-W\left(g^{e}\right)\right]+\left[W(g)-W\left(g^{r}\right)\right] .
$$

Choosing the regular network $g^{r}$ such that $D\left(g^{r}\right)=D(g)$, the first summand contains the contribution to welfare from the pure density in a network, as $\eta_{i}\left(g^{\prime}\right)=\eta_{j}\left(g^{\prime}\right)$ for any $i, j \in N$ and $g^{\prime} \in\left\{g^{e}, g^{r}\right\}$. The welfare effects from the dispersion of collaboration ties among the firms are, therefore, completely captured by the second summand. The following result shows that, due to the linear demand system in our model, we can go a step further and measure the dispersion effect by another simple statistic of the degree distribution.

Lemma 2. The total surplus and the consumer surplus in network $g$ can be written as functions of the density and the normalized degree variance. In particular, $W(g)=Y[D(g), C(g)]$ with:

$Y[D(g), C(g)]=Y[0,0]+\left(\phi_{1}+\phi_{2} D(g)-\frac{n(n-1)}{2} f\right) D(g)+\phi_{3} C(g)$,

where $Y[0,0]>0, \phi_{1}>0, \phi_{2}>0$, and $\phi_{3} \geq 0$. 
The lemma states that the welfare-relevant properties of a network are completely captured by its density and its (normalized) degree variance. The proof and the precise specification of the parameters are given in the Appendix. As is shown in equation (5), the contribution to welfare from the network density can be positive or negative depending on whether or not the linking costs exceed the social returns to a marginal cost reduction. The density enters the welfare function quadratically $\left(\phi_{2}>0\right)$, because every additional collaborative tie increases the effective market size, thereby generating a larger accessible surplus for firms and consumers. Moreover, because $\phi_{3}$ is typically strictly larger zero, it follows that, having fixed the density, social welfare increases with the concentration of links in a network.

Proposition 2. Suppose that either the measure of welfare is the total surplus or $\beta \in(0,1)$. An efficient network $g^{*}$ is maximally concentrated among the networks with the same density: if $D\left(g^{*}\right)=D$ for any $0<D<1$, then $C\left(g^{*}\right)=\max \{C(g) \mid D(g)=D\}$.

The implications for the structure of an efficient network can be illustrated with the help of Figure 1. The proposition implies that the inter-linked star network in the figure attains a higher welfare than the ring, but also that the star is more efficient than the dominant group. This suggests that, from an efficiency point of view, it is desirable to maximally concentrate a given number of cost-reducing links around the smallest number of firms possible.

This result is surprising for several reasons. The first thing to note is that the proposition applies to a wide range of market settings and is independent of whether welfare is measured in terms of consumer surplus or total surplus. In fact, there is only one case contained in our model, in which social welfare does not increase in link concentration. ${ }^{10}$ Second, it is interesting to notice that the proposition opts for a corner solution, where the only constraint to concentration is the maximum degree that a group of firms can attain in a network of a given density. Such a solution may not be so surprising for a market, where products are homogeneous and prices are set by a social planner, because the concentration of collaborative ties enables the planner to reduce the production costs at his low-cost plant. However, as the firms in our model are free to raise their prices above unit production costs, concentration

\footnotetext{
${ }^{10}$ As shown in the Appendix, it is $\phi_{3}=0$ if and only if firms compete a la Cournot with homogeneous products and welfare is measured in terms of consumer surplus. That the distribution of marginal production costs is irrelevant in this case is well known in the literature (Bergstrom and Varian, 1985).
} 
is aligned with the exertion of market power. In this light, Proposition 2 suggests that, if it is for the purpose of concentrating some cost-reducing collaborative ties, concentration in the product market is a tolerable side effect. $^{11}$

The first part of the explanation is that the concentration in a network can be altered by rearranging some of its links, which is a linking cost-neutral transformation of the network structure. The result bases, therefore, solely on the effects of concentration on the distribution of marginal production costs and, in turn, on gross industry profits and consumer surplus. Not surprisingly, the concentration of links has a positive impact on industry profits, because the overall level of market power increases. More interesting is that consumers benefit from concentration as well, which is particularly surprising considering that the firms sell differentiated products in our model.

An intuition can be gained from the following: consider a regular and incomplete network, $g^{r}$, where the unit production costs are given by $c_{i}=c_{j}$ and prices by $p_{i}=p_{j}$ for any $i, j \in N$. A consumer with utility function (1) splits his total consumption into equal parts. Suppose now that link $i j$ is removed and firm $i$ is connected to another firm $k$ instead. In this way, concentration in the network increases and, given the products of the firms are differentiated, $p_{j}$ increases and $p_{k}$ declines. Under quantity competition, for example,

$$
d p_{j}=\gamma\left(1-\nu_{q}-\xi_{q}\right)=\frac{1-\beta}{2-\beta}=-d p_{k}>0
$$

for any $\beta \in(0,1)$. In the more concentrated network, the consumer can still afford the old bundle of goods, because prices have changed at the same absolute rate. However, he can save costs and still obtain the same utility by shifting some of his consumption from good $j$ to $k$. Hence, increasing network concentration yields cost savings for the consumer. Moreover, because the demand for good $k$ has increased, a subsequent price reduction of $p_{k}$, compensated by a price increase of another good, is even more favorable than the previous one. Thus, any subsequent increase in network concentration leads to further cost savings, where the rate of savings is rising with every link rewired. This shows that, even in a market with differentiated products,

\footnotetext{
${ }^{11}$ In fact, it can be shown for our model that, within the class of networks with a fixed density, the normalized degree variance is proportional to the Herfindahl index of product market concentration.
} 
it can be desirable to maximally concentrate a given number of collaboration ties around a small group of firms.

Efficient networks and linking costs. In the following, we characterize the structure of the efficient network in a comparative static analysis, where we focus on the role of linking costs. First, a general result for industries with an arbitrary number of firms is provided. Because a characterization of the precise network structure is complicated in this case, we revert to an analysis of the range of linking costs for which the efficient network exhibits at least some concentration. A detailed characterization is provided later in the section, where we assume some specific numbers of firms.

The following is our general result for markets with $n>2$.

Proposition 3. Suppose the firms sell sufficiently close substitutes $(\beta>\tilde{\beta})$. Suppose, moreover, that the measure of welfare is the total surplus (under price or quantity competition) or the consumer surplus under price competition. There exist $f_{1}$ and $f_{2}$, with $0<f_{1}<f_{2}$, such that in the efficient network, $C\left(g^{*}\right)>0$ if and only if $f \in\left(f_{1}, f_{2}\right) .{ }^{12}$

The proof is deferred to the supplementary material to this article published on the $R J E$ website. In the proof, we exploit the fact that a particular concentrated network, namely the star, dominates the empty and the complete network in terms of welfare, if linking costs are on an intermediate level and the firms sell sufficiently similar products. The proposition confirms the findings of Goyal and Joshi (2003), who show that the efficient network is the complete network, when the costs of linking are small. Moreover, it is in line with the intuition that the efficient network is empty for large linking costs. However, the main insight is that for intermediate linking costs the efficient network is characterized by a concentration of collaborative ties.

The following examples fill the gaps in Proposition 3 by adding three further insights: first of all, the proposition is not clear about the precise structure of the efficient network for intermediate linking costs. The examples provide a detailed characterization. Second, it is yet not clear whether a concentrated network might be efficient for small $\beta$ as well. In the first example, concentration is not desirable, when firms sell weak substitutes $(\beta \leq \tilde{\beta})$. In light of the discussion following Proposition 1, this is not very surprising.

\footnotetext{
${ }^{12} \mathrm{~A}$ concentrated network can also maximize the consumer surplus under quantity competition (see Example 2 below). This, however, requires $\tilde{\beta}<\beta<\hat{\beta}$.
} 
The reason is that the welfare function needs to be sufficiently submodular in order to obtain a concentrated efficient architecture. Yet, the strength of submodularity increases with the intensity of product market competition. Finally, the proposition does not comprise the case of the consumer surplusmaximizing network under quantity competition. The second example shows that the efficient network may be concentrated even in this case. ${ }^{13}$

Example 1. Suppose that $n=3$ and moreover that the firms compete in prices and welfare is measured in terms of total surplus. Let $\beta \leq 0.62$. Then, $g^{*}=g^{c}$, if $f \leq f_{2}$, and $g^{*}=g^{e}$ otherwise. Moreover, let $\beta>0.62$. Then: ${ }^{14}$

$$
\left(g^{*}, D\left(g^{*}\right), C\left(g^{*}\right)\right)= \begin{cases}\left(g^{c}, 1,0\right) & \text { if } 0 \leq f \leq f_{1} \\ \left.g^{s}, 2 / 3,1\right) & \text { if } f_{1} \leq f \leq f_{2} \\ \left.g^{n^{\prime}}, 1 / 3,1\right) & \text { if } f_{2} \leq f \leq f_{3} \\ \left.g^{e}, 0,0\right) & \text { if } f_{3} \leq f\end{cases}
$$

The following example characterizes the efficient network in the limit case of market with a continuum of firms. For this purpose, let us depict the effective market size in a large market by a parameter $A$, where $A>$ 1 , and the costs of link formation by $\rho$. We obtain the following network characterization.

Example 2. Suppose that $n \rightarrow \infty$ and moreover that the firms compete in quantities and the welfare measure is the consumer surplus. Let $\beta=0.5$, $\gamma=1$, and $A=2$. Then: ${ }^{15}\left(g^{*}, D\left(g^{*}\right), C\left(g^{*}\right)\right) \approx$

$$
\begin{cases}\left(g^{c}, 1,0\right) & \text { if } 0 \leq \rho \leq 2.4 \\ \left(g^{n^{\prime}}, 5.6-1.9 \rho, 1-20(\rho-2.7)^{2}\right) & \text { if } 2.4 \leq \rho \leq 2.7 \\ \left(g^{x}, 10.1-3.5 \rho, 1-20(\rho-2.7)^{2}\right) & \text { if } 2.7 \leq \rho \leq 2.9 \\ \left(g^{e}, 0,0\right) & \text { if } 2.9 \leq \rho\end{cases}
$$

\footnotetext{
${ }^{13}$ Due to space constraints, we only briefly summarize the examples here. More details on their derivation can be found in the supplementary material on the $R J E$ website (http://www.rje.org/sup-mat.html).

${ }^{14}$ The critical cost levels are given by $f_{1}=\frac{(3-\beta)(1+\beta)\left(3 \alpha-3 \gamma_{0}+\gamma\right) \gamma}{6(1+2 \beta)}+\frac{(3+5 \beta)(1+\beta) \gamma^{2}}{3(2+3 \beta)^{2}(1-\beta)}, f_{2}=$ $\frac{(3-\beta)(1+\beta)\left(\alpha-\gamma_{0}+\gamma\right) \gamma}{2(1+2 \beta)}$, and $f_{3}=\frac{(3-\beta)(1+\beta)\left(3 \alpha-3 \gamma_{0}+5 \gamma\right) \gamma}{6(1+2 \beta)}-\frac{(3+5 \beta)(1+\beta) \gamma^{2}}{3(2+3 \beta)^{2}(1-\beta)}$.

${ }^{15}$ The parameter specification is not necessary to obtain some closed-form expressions for the density and degree variance in the efficient network, but the unspecified versions are too lengthy to be presented here.
} 
Thus, both examples suggest that the efficient network has a highly concentrated structure, if linking costs are on intermediate levels.

To summarize the section, we have seen that an efficient network of costreducing ties is characterized by a concentrated network structure in a wide range of market settings. In particular, concentration is efficient, when linking costs are on an intermediate level and competition in the product market is sufficiently intense. Despite the fact that concentrated networks facilitate the exertion of market power, the reason for their efficiency is that they enable cost-efficient production of the market output.

\section{Match of stable and efficient networks}

In this section, we try to relate the previous findings to the structures of inter-firm alliance networks in the real world. At first sight, our findings put the observed, highly concentrated structures of several high-tech industries in a rather positive light. However, because our analysis suggests that concentration should be maximal for a given number of links, it might still be that the observed networks are not concentrated enough. Moreover, as the efficient network can be empty or complete, they might be too dense or too sparse.

In order to shed some light on this issue, this section compares the structure of the efficient network with the equilibrium predictions of the model. The latter have been exhaustively characterized in Goyal and Joshi (2003, 2006), who investigate two different equilibrium concepts: network stability and network stability against transfers. In the following, we compare the efficient structures with each of these. Because a comparison is difficult for markets with a finite number of firms, the section contains only formal statements for the limit case of a market with a continuum of firms $(n \rightarrow \infty)$. The relevance of the results is discussed at the end of the section. However, let us point out here that due to the focus on markets with differentiated products and asymmetric costs the analysis of the limit case does not constitute a breach with our fundamental premise of strategically interacting firms.

Match with stable networks. Goyal and Joshi (2003) define a stable network as a network, where any firm that is linked to another has no incentive to sever the link, and any two firms that are not linked should have no incentive to establish a collaboration link. Moreover, no firm should have an 
incentive to delete all its links (Goyal and Joshi, 2003). It turns out that this stability concept is very appealing in our context, because it sufficiently restricts the set of equilibrium networks and enables a general characterization of their efficiency. The following statement is a corollary of Proposition 3.1 in Goyal and Joshi (2006) and an immediate implication of the fact that a firm's profit function, as given in (3), satisfies their requirements of convexity with respect to links and market-wide externalities.

Lemma 3. Suppose a large industry $(n \rightarrow \infty)$. A stable network exists and is empty, complete or has a dominant group architecture.

Note that each of the three architectures are also contained in the characterization of the efficient networks in Proposition 1. In particular, one should note that in a dominant group architecture all collaborative ties are concentrated around a subset of the firms. This gives room for the possibility that the individual considerations of firms lead to the formation of an efficient network structure. However, as Figure 1 illustrates for a market with seven firms, a dominant group, $g^{4}$, with four firms in the center does not attain the same high degree variance as a star network of the same density. Thus, as an implication of Proposition 2 architecture $g^{4}$ is inefficient, because welfare can be increased by rearranging some of its links. Furthermore, in some unreported analyses, we constructed networks that have the same density as some dominant group of size $n^{\prime} \geq 4$, but attain a larger degree variance. Even though all these networks had an inter-linked star architecture in common, it was hard to derive a general construction rule that can be applied to arbitrary $n$ and $n^{\prime}$.

In contrast, for a market with a continuum of firms, we can take advantage of the fact that the maximum variance in a network of given density can be approximated by a simple expression. Formally, let $\hat{C}(D, n)$ depict the maximum (normalized) degree variance, $\hat{C}(D, n)=\max \{C(g) \mid D(g)=$ $\left.D, n=n^{\prime}\right\}$. As we derive in the Appendix, it is:

$$
\lim _{n \rightarrow \infty} \hat{C}(D, n)=\frac{256}{27} \max \left\{D^{\frac{3}{2}}(1-\sqrt{D}),(1-D)^{\frac{3}{2}}(1-\sqrt{1-D})\right\} .
$$

Comparing the expression with the variance in a dominant group architecture (which is the first expression in the brackets) enables a welfare assessment of stable networks. 


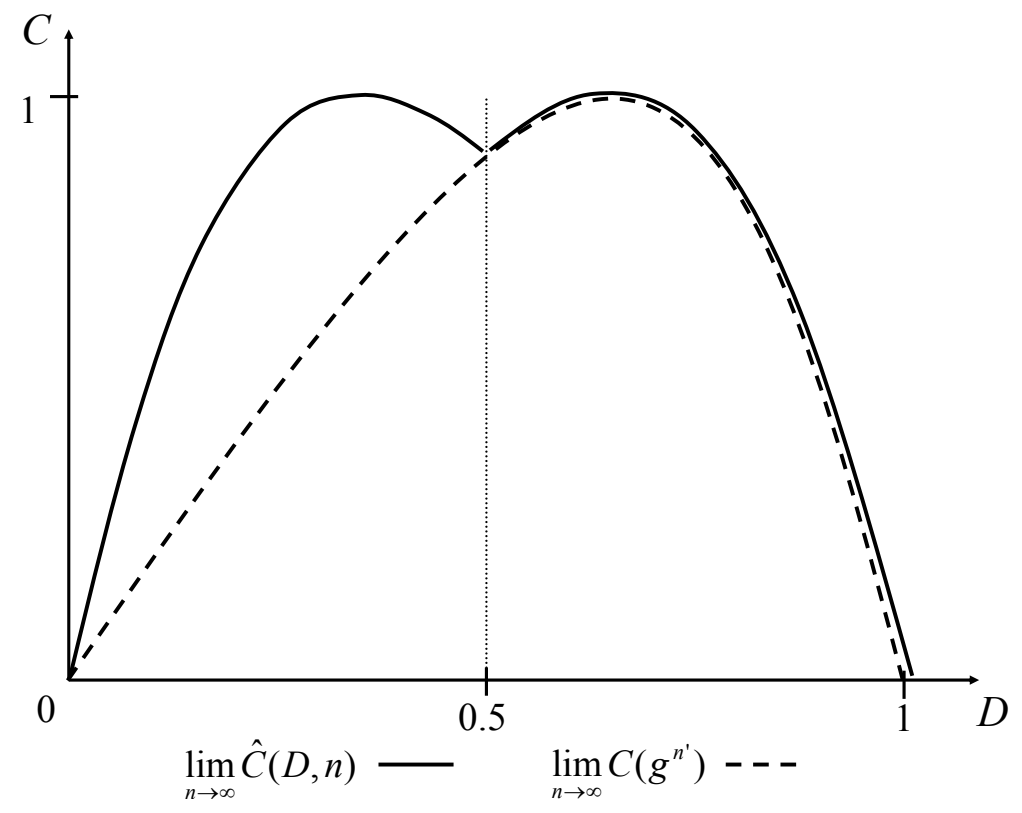

Figure 2: Network concentration in a large industry as a function of density

Proposition 4. Suppose a large industry $(n \rightarrow \infty)$. Suppose moreover that either the measure of welfare is the total surplus or $\beta \in(0,1)$. Every stable network of density $0<D(g)<0.5$ is inefficient.

Proof. The proof follows from Lemmas 2 and 3 in combination with Figure 2 , which plots the normalized degree variances of a dominant group architecture $g^{n^{\prime}}$ (depicted by the dashed line) and a maximum-variance network as functions of density.

The result states that any dominant group architecture consisting of less than half of the maximal attainable links is inefficient. The reason is highlighted in Figure 2 which shows that, for each of these networks, there exists a network of the same density that attains a larger degree variance and is, therefore, more efficient. Hence, social welfare can be increased in a dominant group architecture by rewiring some of the ties of the group of connected firms. Which collaborative ties should replace the links of the connected firms? Proposition 1 provides the answer. From the proposition, it follows that a sparse maximum-variance network must have an inter-linked star architecture. This suggests that the rewired ties should connect a subset of 
the group of connected firms with some of the firms that are isolated in a dominant group architecture.

Despite the clear assertion concerning the sub-optimality of the sparse stable networks, Proposition 4 leaves open the question of whether the efficient network is indeed more concentrated or whether it is denser or sparser. Moreover, the proposition does not allow for a welfare assessment of the dense dominant groups, because these architectures already attain maximal degree variance. Can a dense dominant group be stable and efficient at the same time? It turns out that the answers to both issues depend on the precise cost and demand parameters and, as is shown in an accompanying paper to this article, in particular on the degree of product substitutability (Westbrock, 2008). For low degree of substitutability ( small $\beta$ ), every sparse and stable dominant group architecture consists of too few links, whereas every sparse dominant group is too dense when products are close substitutes. Finally, for intermediate levels of $\beta$, a sparse dominant group can be of proper density, but it is certainly too little concentrated. Even though the variation of $\beta$ produces comparable tensions between the efficient network and the dense and stable networks, the important difference is that for any $0<\beta<1$ there exists a dense and stable dominant group architecture that is efficient at the same time.

Match with transfer-stable networks. Let us turn to a comparison between the efficient networks and the stable networks, when transfer payments between firms are permitted. Motivated by the analysis in Goyal and Joshi (2003), we suspect that transfers might mitigate the discrepancy outlined above. As the authors conclude, dominant group architectures are stable without transfers, because the isolated firms are reluctant to form some collaboration links with the connected firms. Hence, transfers might enable the latter to sustain some additional links with the isolated firms and, therefore, increase concentration in equilibrium. In fact, the authors show that transfer-stable networks may have highly concentrated inter-linked star architectures.

In the following analysis, we apply Goyal and Joshi's (2003) criterion of stability against transfers. The question is whether a sparse efficient network with an inter-linked star architecture can be supported in equilibrium of a large industry. 
Proposition 5. Suppose a large industry $(n \rightarrow \infty)$. Every transfer-stable network of density $0<D(g)<0.5$ is inefficient.

The proof is provided in the Appendix. The result shows that, in contrast to our expectations, transfers are not sufficient to sustain the desired network structure. The reason is that in an inter-linked star architecture there are a periphery of firms that supply the central firms with some additional costreducing ties. However, as shown in the proof, there does not exist any transfer payment satisfying the incentive compatibility constraint of a central firm to sponsor such a tie, encouraging at the same time a peripheral firm to propose it.

We conclude this section by observing that our attempt to reconcile strategically stable and efficient networks results in an overall negative picture. Even though a stable network might be efficient, if it is sufficiently dense, our analysis of the sparse networks shows that the desired, highly concentrated inter-linked star architectures are not incentive compatible. The importance of the analysis in this section rests on the fact that sparse alliance networks between a large number of firms are a typical phenomenon in many high-tech industries. The network in the biotech and pharmaceutical industry of the 1990s, for example, consisted of about 1, 400 companies and had a density of just $0.002 .{ }^{16}$ Similar, Duysters and Vanhaverbeke (1996) report for the DRAM industry of the 1980s a total of 72 suppliers and a density of 0.07 . These observations suggest that the networks characterized in Propositions 4 and 5 are of high empirical relevance.

\section{Robustness}

So far, we have disregarded some of the issues that might violate the favorable assessment of highly concentrated networks. Here, we briefly discuss the relationship of each of these issues to our results on the structure of the efficient network.

Market exit. According to Assumption 1, no firm is forced out of the market by occupying only a peripheral position in the network and thereby having a comparative cost disadvantage. However, market exit of a peripheral

\footnotetext{
${ }^{16}$ The figures are calculated on base of Figure 3 in Powell, Koput, White, and OwenSmith (2005).
} 
firm can be detrimental, because (1) competition in the product market is reduced, and (2) the central firms lose a valuable collaboration partner.

In the supplementary material to this article, we provide an example in which the benefits of a concentrated network outweigh both of these concerns. The underlying intuition is that, even in the model of Section 3, the cost disadvantage of a peripheral firm may be so strong that it sells only an insignificant small quantity in the market. Hence, from a welfare perspective it is only a marginal loss, if this firm exits the market altogether. Moreover, not every lost collaboration tie is necessarily detrimental. On the contrary, Goyal and Moraga-Gonzáles (2001) show that competing firms might have an incentive to form excessively many ties in order to seize their rivals' market shares.

Convex linking costs. The model in this article is simple as compared to other studies on R\&D collaboration by assuming linear demands and costs (e.g., Katz, 1986; Leahy and Neary, 1997). Yet, a concave demand schedule or convex costs might erode the favorable assessment of highly concentrated networks. Here, we will briefly discuss the robustness of Propositions 1 and 2 with respect to the simplest modification in this direction: convex linking costs. Let us therefore violate Assumption 2 by assuming $F_{i}(g)=\frac{1}{2} f \eta_{i}(g)^{2}$, instead. Then, if:

$$
W(g+i j+i k)-W(g+i k)-f \eta_{j}-f>W(g+i k)-W(g)-f \eta_{k}
$$

is satisfied for any $g$ with $\eta_{j}(g) \geq \eta_{k}(g)+1$, Lemma 1 applies. Moreover, the cost term in welfare function (5) is replaced by $\frac{n}{2} f\left((n-1)^{2} D(g)^{2}+V(g)\right)$. Hence, as long as $f$ is sufficiently small as compared to the social benefits from concentration, both Propositions 1 and 2 carry over to a model with convex linking costs. Otherwise, if $\frac{n}{2} \hat{V}(n) f>\phi_{3}$, a network with an an equal dispersion of links is more desirable.

Spillovers. The focus of a large body of the literature on research collaboration is the effect of knowledge spillovers to the whole industry (e.g., d'Aspremont and Jacquemin, 1988) or via the links in a network (Goyal and Moraga-Gonzáles, 2001). A central finding is that the efficient market structure of collaborative agreements depends on the nature and the size of these spillovers. However, Assumption 3 excludes spillovers from the model in this 
article. A modification in this direction is the following. Suppose a firm's marginal production cost in network $g$ is given by:

$$
c_{i}(g)=\gamma_{0}-\gamma\left[(1-\theta) \eta_{i}(g)+(\theta-\vartheta) \sum_{j \in N_{i}(g)} \eta_{j}(g)+\vartheta \sum_{k \in N} \eta_{k}(g)\right]
$$

where $0<\vartheta \leq \theta<1$. Hence, costs are additionally reduced by the collaborative links of firm $i$ 's neighbors at a rate $\theta$ and by the links of all other firms in the network at a rate $\vartheta$. For a market with Cournot competition and homogeneous products, we have checked the robustness of Propositions 1 and 2 with respect to a modification towards industry-wide spillovers $(\theta=\vartheta)$. The analysis is presented in the supplementary material to this article. It turns out that both results carry over, if the rate of spillover is sufficiently small. In contrast, our attempt to generalize the results to situations with network-dependent spillovers $(\theta>\vartheta)$ has not been very fruitful. The reason is that, in this case, the social benefits to a single link or to a whole network structure depend on richer network properties than the ones required in Lemmas 1 and 2.

\section{Conclusion}

In his seminal article, Arrow (1962) points to the imperfections aligned with the demand and the production of information, and to the consequences for the efficient market structure in innovating industries. This article investigates the implications for the structure of the efficient R\&D collaboration network in the model of Goyal and Joshi (2003).

A first important result is that the efficient network typically has a dominant group or an inter-linked star architecture, both of which are highly asymmetric network structures. Second, our analysis shows that it is optimal to maximally concentrate all collaborative activities around a subset of the firms in a network. Both findings follow from a fundamental property of the demand for research output: the indivisibility in its use makes the accumulation of costly joint research at a small group of firms efficient. Hence, in the light of Goyal and Joshi's (2003) characterization of the strategically stable networks, which are asymmetric as well, our analysis suggests that concentration is a natural characteristic of a collaboration network between firms. 
The article also provides a comparison of stable and efficient networks, where the focus is on the question of whether an efficient structure can be supported in equilibrium. Our findings are unambiguous and negative for the networks of low or moderate density: no sufficiently sparse network that is efficient, is strategically stable at the same time. The reason for this failure in the network structure lies in the individual considerations of the firms. A sparse efficient network has an inter-linked star architecture, which consists of some collaborative ties between the firms in the center and the periphery of the network. Yet, our analysis shows that the peripheral firms are reluctant to sustain these links.

Our findings might have some relevance for policy programs to foster collaborative activity, like the EU Framework Programmes on R\&D. A general implication from the analysis in this article is that a sensible program should take into account the structural properties of the network of ongoing collaborations between firms. In particular, our analysis suggests that a program should aim at expanding the ties of the already actively collaborating firms. Moreover, as the findings from our comparison between stable and efficient networks imply, this should be achieved by encouraging collaborative projects between the active collaborators and the smaller and younger enterprises at the periphery of the network.

As a caveat to our normative conclusions, let us remark that there are some restrictive assumptions underlying the model in this article. We have confined our analysis to collaborative ties between firms bearing an incremental innovation, meaning that a link aims to improve the current products of the firms. However, collaborative ties may also enable drastic innovations that replace old generations of products. The welfare assessment of asymmetric network structures is a completely open issue in this context. Another issue, which has only briefly been addressed in this study, is the assessment of asymmetric networks under market exit and network-dependent spillovers. 


\section{Appendix}

The Appendix contains the proofs of Lemmas 1 and 2 and Propositions 1 and 5. Moreover, we determine the normalized degree variance (used in Proposition 4) and the architecture (used in Proposition 5) of an efficient network in a large industry.

Proof of Lemma 1. Given that the product market is in equilibrium, let us write $q_{i}(g)$ to denote the equilibrium quantity of any firm $i$ in network $g$. Moreover, let the term $\Delta q_{u}^{i j}$ depict the change in equilibrium quantity of firm $u$ upon the formation of link $i j$. Thus, for $u \in\{i, j\}$, it is $\Delta q_{u}^{i j}=-\gamma\left(\frac{\partial q_{i}}{\partial c_{i}}+\frac{\partial q_{i}}{\partial c_{j}}\right)$, whereas for $u \in N \backslash\{i, j\}, \Delta q_{u}^{i j}=-2 \gamma \frac{\partial q_{i}}{\partial c_{j}}$. In the supplementary material to this article (http://www.rje.org/sup-mat.html), it is shown that for any network $g$ with $i j, i k \notin g$, one can write:

$$
\begin{aligned}
& \sum_{i \in N}\left[\Pi_{i}(g+i j+i k)-2 \Pi_{i}(g+i k)+\Pi_{i}(g)\right]= \\
& 2 \lambda\left[\Delta q_{i}^{i j} \Delta q_{i}^{i k}+\sum_{u \in N \backslash\{i, j, k\}} \Delta q_{u}^{i j} \Delta q_{u}^{i k}+\Delta q_{j}^{i j}\left(q_{j}(g+i k)-q_{k}(g)\right)\right. \\
& \left.+\Delta q_{k}^{i j}\left(q_{k}(g+i k)-q_{j}(g)\right)\right] \\
& U(g+i j+i k)-2 U(g+i k)+U(g)= \\
& \lambda\left[\Delta q_{i}^{i j}\left(\frac{\gamma}{\lambda}-\Delta q_{i}^{i k}\right)-\sum_{u \in N \backslash\{i, j, k\}} \Delta q_{u}^{i j} \Delta q_{u}^{i k}+\left(\frac{\gamma}{\lambda}-\Delta q_{j}^{i j}\right)\left(q_{j}(g+i k)-q_{k}(g)\right)\right. \\
& \left.-\Delta q_{k}^{i j}\left(q_{k}(g+i k)-q_{j}(g)\right)\right] \text {. }
\end{aligned}
$$

If $\eta_{j}(g)=\eta_{k}(g)+1$ the expressions simplify to:

$$
\begin{aligned}
& \sum_{i \in N}\left[\Pi_{i}(g+i j+i k)-2 \Pi_{i}(g+i k)+\Pi_{i}(g)\right]= \\
& 2 \lambda\left[\sum_{u \in\{i, j\}}\left(\Delta q_{u}^{i j}\right)^{2}+\sum_{v \in N \backslash\{i, j\}}\left(\Delta q_{v}^{i j}\right)^{2}\right] \\
& U(g+i j+i k)-2 U(g+i k)+U(g)= \\
& \quad \lambda\left[\sum_{u \in\{i, j\}} \Delta q_{u}^{i j}\left(\frac{\gamma}{\lambda}-\Delta q_{u}^{i j}\right)-\sum_{v \in N \backslash\{i, j\}}\left(\Delta q_{v}^{i j}\right)^{2}\right] .
\end{aligned}
$$

because then $q_{j}(g+i k)-q_{k}(g)=\Delta q_{j}^{i j}$ and $q_{k}(g+i k)-q_{j}(g)=\Delta q_{j}^{i k}$. Convexity of industry profits follows immediately from the fact that the summands in brackets of (13a) are both positive and $\lambda>0$. Although the terms in brackets of (13b) are of opposite sign, it can be shown that their sum is positive for any $n>2$ and $\beta \in(0,1]$ and regardless of whether firms compete in quantities or in prices. Moreover, it can be verified that if $\eta_{j}(g)>\eta_{k}(g)+1,(12 \mathrm{a})$ is even greater than (13a), and (12b) is greater than (13b), which establishes part (i) of the lemma. (On the other hand, for $\eta_{j}(g)<\eta_{k}(g)+1$, we can find cost and demand parameters for which convexity is not satisfied.) 
We now turn to part (ii) of the lemma. For any network $g$ with $i j, k l \notin g$ and $k, l \in N \backslash\{i, j\}$ we may write:

$$
\begin{aligned}
\sum_{i \in N} & {\left[\Pi_{i}(g+i j+k l)-\Pi_{i}(g+k l)-\Pi_{i}(g+i j)+\Pi_{i}(g)\right]=} \\
2 \lambda\left[\sum_{u \in\{i, j, k, l\}} \Delta q_{u}^{i j} \Delta q_{u}^{k l}+\sum_{v \in N \backslash\{i, j, k, l\}} \Delta q_{v}^{i j} \Delta q_{v}^{k l}\right] & (1, U(g)= \\
U(g)+k l)-U(g+k l)-U(g+i j)+U(g)= & \quad \lambda\left[\sum_{u \in\{i, j\}}\left(\frac{\gamma}{\lambda}-\Delta q_{u}^{i j}\right) \Delta q_{u}^{k l}-\sum_{v \in\{k, l\}} \Delta q_{v}^{i j} \Delta q_{v}^{k l}-\sum_{w \in N \backslash\{i, j, k, l\}} \Delta q_{w}^{i j} \Delta q_{w}^{k l}\right] .
\end{aligned}
$$

It can easily be verified that the expressions in brackets of (14a) are negative for any $n>2$ and $\beta \in(0,1]$, and regardless of whether we consider price or quantity competition in the market. The term in brackets of $(14 \mathrm{~b})$ equals under quantity competition $-4 \beta^{2} \frac{n-2-(n-1) \beta}{(2+(n-1) \beta)^{2}(2-\beta)^{2}}$, which is smaller zero if and only if $n>2$ and $\beta<\frac{n-2}{n-1}$. Under price competition, negativity requires again only $n>2$ and $\beta \in(0,1)$.

Proof of Proposition 1. We first characterize the regular and efficient architectures. Suppose a regular network $g$ that is neither empty nor complete. Thus, there exist distinct $i, j, k \in N$ such that $i j \in g$ and $i k \notin g$. For efficiency of $g$, it must hold for any $i j \in g$ that $W(g)-W(g-i j) \geq 0$. In network $g-i j$, however, $\eta_{k}(g-i j)=\eta_{j}(g-i j)+1$ and therefore, from convexity in links, $W(g+i k)-W(g)>W(g)-W(g-i j)$. This implies a contradiction to network $g$ having an efficient architecture. Hence, if the efficient network is regular it must either be the empty or the complete network.

Let us turn to a characterization of irregular architectures. Suppose first that an efficient and irregular network $g^{*}$ induces a two-point degree partition, $\left\{h_{0}, h_{m}\right\}$, and suppose $\left|h_{m}\right|=2$. Then we have a dominant group architecture. Assume next a network $g$ that induces a degree partition, $\left\{h_{0}, h_{m}\right\}$, with $\left|h_{m}\right|>2$, and in which for every $i \in h_{m}$, $\eta_{i}(g)<\left|h_{m}\right|-1$. Hence, there exist distinct $i, j, k \in h_{m}$ with $i k \in g$ and $i j \notin g$. For $g$ having an efficient architecture, it must be $W(g)-W(g-i k) \geq 0$. However, $\eta_{j}(g-i k)=$ $\eta_{k}(g-i k)+1$. Hence, convexity in links applies and therefore $W(g+i j)-W(g)>$ $W(g)-W(g-i k)$. This implies a contradiction. Thus, if an efficient network $g^{*}$ induces a two-point degree partition with $\left|h_{m}\right|>2$, then $\eta_{i}\left(g^{*}\right)=\left|h_{m}\right|-1$ for every $i \in h_{m}$. This is a dominant group architecture.

Suppose next that a network $g^{*}$ with an efficient architecture induces a degree partition, $\left\{h_{l_{1}}, h_{l_{2}}, \ldots, h_{m}\right\}$, with at least two groups of firms with a positive degree. We show that for every firm $i$ with $\eta_{i}\left(g^{*}\right)>0$ it must be $i j \in g^{*}$, if $j \in h_{m}$. Suppose not, then we have a network $g$ that induces a degree partition with more than one group of positive degree, where there are distinct $i, j \in N$ such that $\eta_{i}(g)>0$ and $j \in h_{m}$ but $i j \notin g$. Because $\eta_{i}(g)>0$, there must exist a firm $k \in N$ with $i k \in g$. However, as $\eta_{k}(g-i k) \leq \eta_{j}(g-i k)-1$, convexity in links applies and, therefore, $W(g+i j)-W(g)>W(g)-W(g-i k)$. This implies a contradiction. Hence, in an efficient network $g^{*}$, it must be for any $j \in h_{m}$ that $i j \in g^{*}$ for every firm $i$ with $\eta_{i}\left(g^{*}\right)>0$. This is an inter-linked star. 
Proof of Lemma 2. We will show that one may write:

$$
\begin{aligned}
U(g) & =U\left(g^{e}\right)+\varphi_{1} D(g)+\varphi_{2} D(g)^{2}+\varphi_{3} C(g) \\
\sum_{i \in N} \Pi_{i}(g) & =\sum_{i \in N} \Pi_{i}\left(g^{e}\right)+\varphi_{4} D(g)+\varphi_{5} D(g)^{2}-\frac{1}{2} f n(n-1) D(g)+\varphi_{6} C(g)
\end{aligned}
$$

where $\varphi_{1}$ to $\varphi_{6}$ are defined below. The claim follows immediately from this. In particular, if $Y[D, C]$ measures the consumer surplus it is $Y[0,0]=U\left(g^{e}\right), \phi_{1}=\varphi_{1}, \phi_{2}=\varphi_{2}$ and $\phi_{3}=\varphi_{3}$, whereas if welfare is measured in terms of total surplus:

$$
Y[0,0]=U\left(g^{e}\right)+\sum_{i \in N} \Pi_{i}\left(g^{e}\right), \quad \phi_{1}=\varphi_{1}+\varphi_{4}, \quad \phi_{2}=\varphi_{2}+\varphi_{5}, \quad \text { and } \quad \phi_{3}=\varphi_{3}+\varphi_{6} .
$$

Let us begin by decomposing the welfare in network $g$ into:

$$
W(g)-W\left(g^{e}\right)=\left[W(g)-W\left(g^{r}\right)\right]+\left[W\left(g^{r}\right)-W\left(g^{e}\right)\right],
$$

where the regular network $g^{r}$ is such that $D\left(g^{r}\right)=D(g)$. Note moreover that $C\left(g^{r}\right)=$ $C\left(g^{e}\right)=0$.

We derive each summand in turn and begin with $W\left(g^{r}\right)-W\left(g^{e}\right)$. Using the fact that in equilibrium $p_{i}=\lambda q_{i}+c_{i}$, we can write for utility and gross industry profits:

$$
\begin{aligned}
U(g) & =\sum_{i \in N}\left(\alpha-c_{i}(g)\right) q_{i}(g)-\left(\lambda+\frac{1}{2}\right) q_{i}(g)^{2}-\frac{\beta}{2} \sum_{j \neq i} q_{i}(g) q_{j}(g) \\
\sum_{i \in N} \pi_{i}(g) & =\lambda \sum_{i \in N} q_{i}(g)^{2} .
\end{aligned}
$$

Because in $g^{r}$ it holds $q_{i}\left(g^{r}\right)=q_{j}\left(g^{r}\right)$ and $c_{i}\left(g^{r}\right)=c_{j}\left(g^{r}\right)=\gamma_{0}-\gamma(n-1) D\left(g^{r}\right)$ for any $i, j \in N$, the difference $W\left(g^{r}\right)-W\left(g^{e}\right)$ may be written as:

$$
\begin{aligned}
U\left(g^{r}\right)-U\left(g^{e}\right)= & n\left[\alpha-\gamma_{0}+\gamma(n-1) D\left(g^{r}\right)\right] q_{i}\left(g^{r}\right) \\
& -n\left[\alpha-\gamma_{0}\right] q_{i}\left(g^{e}\right)-n \frac{2 \lambda+1+(n-1) \beta}{2}\left[q_{i}\left(g^{r}\right)^{2}-q_{i}\left(g^{e}\right)^{2}\right] \\
\sum_{i \in N} \Pi_{i}\left(g^{r}\right)-\sum_{i \in N} \Pi_{i}\left(g^{e}\right)= & n \lambda\left[q_{i}\left(g^{r}\right)^{2}-q_{i}\left(g^{e}\right)^{2}\right]-\frac{1}{2} f n(n-1) D\left(g^{r}\right) .
\end{aligned}
$$

Substituting $q_{i}\left(g^{r}\right)^{2}-q_{i}\left(g^{e}\right)^{2}=\left[q_{i}\left(g^{r}\right)-q_{i}\left(g^{e}\right)\right]\left[q_{i}\left(g^{r}\right)-q_{i}\left(g^{e}\right)+2 q_{i}\left(g^{e}\right)\right]$, we obtain:

$$
\begin{aligned}
U\left(g^{r}\right)-U\left(g^{e}\right)= & \gamma n(n-1) D\left(g^{r}\right) q_{i}\left(g^{e}\right) \\
& +n\left[\alpha-\gamma_{0}+\gamma(n-1) D\left(g^{r}\right)\right]\left[q_{i}\left(g^{r}\right)-q_{i}\left(g^{e}\right)\right] \\
& -n \frac{2 \lambda+1+(n-1) \beta}{2}\left[q_{i}\left(g^{r}\right)-q_{i}\left(g^{e}\right)\right]\left[q_{i}\left(g^{r}\right)-q_{i}\left(g^{e}\right)+2 q_{i}\left(g^{e}\right)\right] \\
\sum_{i \in N} \Pi_{i}\left(g^{r}\right)-\sum_{i \in N} \Pi_{i}\left(g^{e}\right)= & n \lambda\left[q_{i}\left(g^{r}\right)-q_{i}\left(g^{e}\right)\right]\left[q_{i}\left(g^{r}\right)-q_{i}\left(g^{e}\right)+2 q_{i}\left(g^{e}\right)\right] \\
& -\frac{1}{2} f n(n-1) D\left(g^{r}\right) .
\end{aligned}
$$


It can be verified that:

$$
\begin{aligned}
q_{i}\left(g^{r}\right)-q_{i}\left(g^{e}\right) & =(n-1) D\left(g^{r}\right)\left[\Delta q_{i}^{i j}+\left(\frac{n}{2}-1\right) \Delta q_{i}^{j k}\right] \text { and } \\
q_{i}\left(g^{e}\right) & =\frac{\alpha-\gamma_{0}}{\gamma}\left[\Delta q_{i}^{i j}+\left(\frac{n}{2}-1\right) \Delta q_{i}^{j k}\right]
\end{aligned}
$$

where the terms $\Delta q_{i}^{i j}$ and $\Delta q_{i}^{j k}$ are defined in the proof of Lemma 1. Concerning the left-hand term, for example, note that in $g^{r}$ a typical firm is involved in $(n-1) D\left(g^{r}\right)$ links more than in $g^{e}$, and the remaining firms, $j \in N \backslash\{i\}$, are involved in $\left(\frac{n}{2}-1\right)(n-1) D\left(g^{r}\right)$ more links. Upon substitution we can simplify the expressions to:

$$
\begin{aligned}
U\left(g^{r}\right)-U\left(g^{e}\right) & =\varphi_{1} D\left(g^{r}\right)+\varphi_{2} D\left(g^{r}\right)^{2} \\
\sum_{i \in N} \Pi_{i}\left(g^{r}\right)-\sum_{i \in N} \Pi_{i}\left(g^{e}\right) & =\varphi_{4} D\left(g^{r}\right)+\varphi_{5} D\left(g^{r}\right)^{2}-\frac{1}{2} f n(n-1) D\left(g^{r}\right)
\end{aligned}
$$

where

$$
\begin{aligned}
\varphi_{1} & =n(n-1)\left(\alpha-\gamma_{0}\right)\left[\Delta q_{i}^{i j}+\left(\frac{n}{2}-1\right) \Delta q_{i}^{j k}\right] \frac{2 \gamma-[2 \lambda+1+(n-1) \beta]\left[\Delta q_{i}^{i j}+\left(\frac{n}{2}-1\right) \Delta q_{i}^{j k}\right]}{\gamma} \\
\varphi_{2} & =n(n-1)\left[\Delta q_{i}^{i j}+\left(\frac{n}{2}-1\right) \Delta q_{i}^{j k}\right] \frac{2 \gamma-[2 \lambda+1+(n-1) \beta]\left[\Delta q_{i}^{i j}+\left(\frac{n}{2}-1\right) \Delta q_{i}^{j k}\right]}{2} \\
\varphi_{4} & =2 n(n-1)\left(\alpha-\gamma_{0}\right) \lambda \frac{\left[\Delta q_{i}^{i j}+\left(\frac{n}{2}-1\right) \Delta q_{i}^{j k}\right]^{2}}{\gamma} \\
\varphi_{5} & =n(n-1) \lambda\left[\Delta q_{i}^{i j}+\left(\frac{n}{2}-1\right) \Delta q_{i}^{j k}\right]^{2} .
\end{aligned}
$$

The reader might check that $\Delta q_{i}^{i j}+\left(\frac{n}{2}-1\right) \Delta q_{i}^{j k}>0$ and $2 \gamma-[2 \lambda+1+(n-1) \beta]\left[\Delta q_{i}^{i j}+\right.$ $\left.\left(\frac{n}{2}-1\right) \Delta q_{i}^{j k}\right]>0$ for any $\beta \in(0,1]$ and $n>2$ and regardless of whether competition is in quantities or in prices. Hence, $\varphi_{1}, \varphi_{2}, \varphi_{4}$, and $\varphi_{5}$ are all greater than zero.

Let us turn to the derivation of $W(g)-W\left(g^{r}\right)$ and start from:

$$
\begin{aligned}
U(g)-U\left(g^{r}\right)= & \sum_{i \in N}\left[\left(\alpha-\gamma_{0}+\gamma \eta_{i}(g)\right) q_{i}(g)\right] \\
& -n\left[\alpha-\gamma_{0}+\gamma(n-1) D\left(g^{r}\right)\right] q_{i}\left(g^{r}\right) \\
& -\left(\lambda+\frac{1}{2}\right) \sum_{i \in N} q_{i}(g)^{2}-\frac{\beta}{2} \sum_{i \in N} \sum_{j \neq i} q_{i}(g) q_{j}(g) \\
& +n \frac{2 \lambda+1+(n-1) \beta}{2} q_{i}\left(g^{r}\right)^{2} \\
\sum_{i \in N} \Pi_{i}(g)-\sum_{i \in N} \Pi_{i}\left(g^{r}\right)= & \lambda \sum_{i \in N}\left[q_{i}(g)^{2}-q_{i}\left(g^{r}\right)^{2}\right] .
\end{aligned}
$$

The following properties help to simplify the expressions. For any $D(g)=D\left(g^{r}\right)$ : 
(i) $q_{i}(g)-q_{i}\left(g^{r}\right)=\left[\Delta q_{i}^{i j}-\Delta q_{i}^{j k}\right]\left[\eta_{i}(g)-\bar{\eta}\left(g^{r}\right)\right]$

(ii) $q_{i}(g) q_{j}(g)-q_{i}\left(g^{r}\right)^{2}=\left[q_{i}(g)-q_{i}\left(g^{r}\right)\right]\left[q_{j}(g)-q_{i}\left(g^{r}\right)\right]+q_{i}\left(g^{r}\right)\left[q_{i}(g)-q_{i}\left(g^{r}\right)+q_{j}(g)-\right.$ $\left.q_{i}\left(g^{r}\right)\right]$

(iii) $\sum_{i \in N}\left[\eta_{i}(g)-\bar{\eta}\left(g^{r}\right)\right]=0$

(iv) $\sum_{i \in N} \eta_{i}(g)\left[\eta_{i}(g)-\bar{\eta}\left(g^{r}\right)\right]=n V(g)$

(v) $\sum_{i \in N} \sum_{j \neq i}\left[\eta_{i}(g)-\bar{\eta}\left(g^{r}\right)\right]=0$

(vi) $\sum_{i \in N} \sum_{j \neq i}\left[\eta_{i}(g)-\bar{\eta}\left(g^{r}\right)\right]\left[\eta_{j}(g)-\bar{\eta}\left(g^{r}\right)\right]=-n V(g)$.

Applying all of them, we obtain:

$$
\begin{aligned}
U(g)-U\left(g^{r}\right) & =\varphi_{3} C(g) \\
\sum_{i \in N} \Pi_{i}(g)-\sum_{i \in N} \Pi_{i}\left(g^{r}\right) & =\varphi_{6} C(g),
\end{aligned}
$$

with

$$
\begin{aligned}
\varphi_{3} & =n \hat{V}(n)\left[\Delta q_{i}^{i j}-\Delta q_{i}^{j k}\right] \frac{2 \gamma-[2 \lambda+1-\beta]\left[\Delta q_{i}^{i j}-\Delta q_{i}^{j k}\right]}{2} \\
\varphi_{6} & =n \lambda \hat{V}(n)\left[\Delta q_{i}^{i j}-\Delta q_{i}^{j k}\right]^{2} .
\end{aligned}
$$

The reader may check that $\Delta q_{i}^{i j}-\Delta q_{i}^{j k}>0$ and $2 \gamma-[2 \lambda+1-\beta]\left[\Delta q_{i}^{i j}-\Delta q_{i}^{j k}\right] \geq 0$ for any $\beta \in(0,1]$ and regardless of the competition mode (the equality holds if and only if the firms compete in quantities and $\beta=1$ ). Thus, $\varphi_{3} \geq 0$ and $\varphi_{6}>0$.

The efficient network in a large industry. In the following, we determine the normalized degree variance and the architecture of an efficient network in a large industry. The normalized degree variance is determined in two steps: first, we replicate a result from Snijders (1981) on the architecture of a variance-maximizing network for given density. Then, we calculate the degree variance of this architecture.

Define the complementary network to network $g$ by $g_{c}=\{i j: i, j \in N, i \neq j, i j \notin g\}$, which has its links exactly where $g$ does not. Note that $D\left(g_{c}\right)=1-D(g)$ and $C\left(g_{c}\right)=C(g)$. Snijders (1981) shows that for any $n>2$ and for any $0<D<1$, a variance-maximizing network $\tilde{g}=\operatorname{argmax}\{C(g) \mid D(g)=D\}$ satisfies the following properties:

Either $\tilde{g}$ has an architecture $g^{\prime}$, or $\tilde{g}_{c}$ has an architecture $g^{\prime \prime}$, where:

(i) $g^{\prime}$ and $g^{\prime \prime}$ both partition the set of firms into a four-point degree partition $\left\{h_{0}, h_{l_{1}}, h_{l_{2}}, h_{l_{3}}\right\}$, with $l_{1}=\left|h_{l_{3}}\right|, \quad l_{2}=\left|h_{l_{2}}\right|+\left|h_{l_{3}}\right|-1$, and $\quad l_{3}=\left|h_{l_{1}}\right|+\left|h_{l_{2}}\right|+\left|h_{l_{3}}\right|-1$.

Moreover, it is $\left|h_{l_{1}}\right|=1$ and:

(iia) in architecture $g^{\prime}$, it is $\left|h_{l_{2}}\right|+\left|h_{l_{3}}\right|$ the largest integer $I_{1}$ with $I_{1}\left(I_{1}-1\right) \leq n(n-1) D$, and

(iib) in architecture $g^{\prime \prime}$, it is $\left|h_{l_{2}}\right|+\left|h_{l_{3}}\right|$ the largest integer $I_{2}$ with $I_{2}\left(I_{2}-1\right) \leq n(n-$ 1) $(1-D)$. 
According to this, either $\tilde{g}$ or its complement $\tilde{g}_{c}$ have a dominant group-like architecture, where the size of the group of connected firms, $\left|h_{l_{2}}\right|+\left|h_{l_{3}}\right|$, is maximal given the density. The remaining links originate from a single peripheral firm in $h_{l_{1}}$ that is connected only to the group of connected firms.

The (normalized) degree variance of the maximally concentrated network is now determined by $C(\tilde{g})=\hat{C}(D, n)=\max \left\{C\left(g^{\prime}\right), C\left(g^{\prime \prime}\right)\right\}$, where:

$C\left(g^{\prime}\right)=\frac{1}{\hat{V}(n)} \frac{\left(\left|h_{l_{3}}\right|-\bar{\eta}\right)^{2}+\left|h_{l_{3}}\right|\left(I_{1}-\bar{\eta}\right)^{2}+\left(I_{1}-\left|h_{l_{3}}\right|\right)\left(I_{1}-1-\bar{\eta}\right)^{2}+\left(n-I_{1}-1\right) \bar{\eta}^{2}}{n}$

and $C\left(g^{\prime \prime}\right)$ is obtained by replacing $I_{1}$ by $I_{2}$ and $\bar{\eta}$ by $(n-1-\bar{\eta})$. If $n$ is a finite number determining $C(\tilde{g})$ is difficult, because it involves a constrained maximization with respect to $I_{1}$ and $I_{2}$. However, for $n \rightarrow \infty$, architecture $g^{\prime}$ has approximately the normalized degree variance of a dominant group $g^{n^{\prime}}$, which is obtained from $g^{\prime}$ by removing the links of the peripheral firm in $h_{l_{1}}$. More precisely, for the dominant group architecture $g^{n^{\prime}}$, with $n^{\prime}=I_{1}$, and for any $0 \leq\left|h_{l_{3}}\right| \leq n^{\prime}<n$, it is:

$$
\lim _{n \rightarrow \infty}\left[C\left(g^{\prime}\right)-C\left(g^{n^{\prime}}\right)\right]=\lim _{n \rightarrow \infty}\left[256\left|h_{l_{3}}\right| \frac{n\left(2 n^{\prime}-1\right)-4 n^{\prime}\left(n^{\prime}-1\right)+\left|h_{l_{3}}\right|(n-4)}{(3 n-2)^{2}(n-2)(3 n+2)}\right]=0 .
$$

Similarly, one can approximate $C\left(g^{\prime \prime}\right)$ by the normalized variance of the sparser dominant group $g^{n^{\prime \prime}}$. We obtain for the maximum variance of a network of density $D$ :

$$
\begin{aligned}
\lim _{n \rightarrow \infty} \hat{C}(D, n)= & \lim \left[\max \left\{C\left(g^{n^{\prime}}\right), C\left(g^{n^{\prime \prime}}\right)\right\}\right] \\
= & \frac{256}{27} \lim \left[\operatorname { m a x } \left\{D\left(\frac{\sqrt{1-8\left|h_{l_{3}}\right|+4 D n(n-1)}-1}{2 n}-D\right),\right.\right. \\
& \left.\left.(1-D)\left(\frac{\sqrt{1-8\left|h_{l_{3}}\right|+4(1-D) n(n-1)}-1}{2 n}-1+D\right)\right\}\right] \\
= & \frac{256}{27} \max \left\{D^{\frac{3}{2}}(1-\sqrt{D}),(1-D)^{\frac{3}{2}}(1-\sqrt{1-D})\right\} .
\end{aligned}
$$

The first equality follows from the approximation described above. To establish the second equality, we rewrite $\lim C\left(g^{n^{\prime}}\right)=\lim \frac{n^{2}}{\hat{V}(n)} \times \lim \frac{1}{n^{2}} V\left(g^{n^{\prime}}\right)$ and solve $\lim \frac{n^{2}}{\hat{V}(n)}=\frac{27}{256}$ and:

$$
\begin{aligned}
\lim \frac{1}{n^{2}} V\left(g^{n^{\prime}}\right) & =\lim \frac{1}{n^{2}} \frac{n^{\prime}\left(n^{\prime}-1-\frac{n^{\prime}\left(n^{\prime}-1\right)}{n}\right)^{2}+\left(n-n^{\prime}\right)\left(\frac{n^{\prime}\left(n^{\prime}-1\right)}{n}\right)^{2}}{n} \\
& =\lim \frac{1}{n^{2}} \frac{n^{\prime}\left(n^{\prime}-1\right)}{n}\left(n^{\prime}-1-\frac{n^{\prime}\left(n^{\prime}-1\right)}{n}\right)=\lim D\left(\frac{n^{\prime}-1}{n}-D\right) .
\end{aligned}
$$

This follows from $D\left(g^{n^{\prime}}\right)=\frac{n^{\prime}\left(n^{\prime}-1\right)}{n(n-1)}=D-\frac{2\left|h_{l_{3}}\right|}{n(n-1)}$, and hence $\lim n^{\prime}\left(n^{\prime}-1\right) / n^{2}=D$. The second equality is now established by replacing $n^{\prime}=\frac{1}{2}+\frac{1}{2} \sqrt{1-8\left|h_{l_{3}}\right|+4 D n(n-1)}$. The third equality follows from noticing that $\lim \sqrt{1-8\left|h_{l_{3}}\right|+4 D n(n-1)} / n$ equals $\lim \sqrt{\left(1-8\left|h_{l_{3}}\right|\right) / n^{2}-4 D / n+4 D}=2 \sqrt{D}$. Note that the final expression for $\lim \hat{C}(D, n)$ 
is continuous in density and differentiable almost everywhere (with the only exception being $D=0.5)$.

Concerning the architecture of the variance-maximizing network, $\tilde{g}$, note that if $D \geq$ 0.5 the maximum of the two values in the final expression for $\lim \hat{C}(D, n)$ is given by $D^{\frac{3}{2}}(1-\sqrt{D})$. Hence, $\tilde{g}$ has (approximately) the architecture of the dominant group, $g^{n^{\prime}}$, described above, where a share of $n^{\prime} / n=\sqrt{D}$ firms is in the group of connected firms. For $D \leq 0.5$, the maximum is $(1-D)^{\frac{3}{2}}(1-\sqrt{1-D})$. As an implication of Proposition $1, \tilde{g}$ must have an inter-linked star architecture. Because the complementary network has the dominant group architecture, $g^{n^{\prime \prime}}$, network $\tilde{g}$ consists of a center group of firms, $h_{c}$, and a single peripheral group, $h_{p}$. Moreover, the periphery of $\tilde{g}$ comprises a share of $\sqrt{1-D}$ of the total of firms and for each $i \in h_{p}, i j \in \tilde{g}$ if and only if $j \in h_{c}$.

In the following, we investigate transfer-stable networks between a large number of firms and present the proof of Proposition 5. Denote by $t_{i}=\left\{t_{i}^{1}, t_{i}^{2}, \ldots, t_{i}^{n}\right\}$ the transfers offered by firm $i$ to other firms, where $t_{i}^{j} \geq 0$ for all $j \in N$ with $i j \in g$, and $t_{i}^{k}=0$ for $k \in N$ with $i k \notin g$. Moreover, denote by $g_{-i}$ the network that is obtained from $g$ by deleting all links of firm $i$. Goyal and Joshi (2003) define network $g$ stable against transfers, if

(i) for all $i j \in g: \pi_{i}(g)+\pi_{j}(g)-f \geq \pi_{i}(g-i j)+\pi_{j}(g-i j)$

(ii) for all $i j \notin g: \pi_{i}(g)+\pi_{j}(g)+f \geq \pi_{i}(g+i j)+\pi_{j}(g+i j)$

(iii) there exist transfers $t_{i}, i \in\{1,2, \ldots, n\}$, such that

$$
\pi_{i}(g)+\sum_{j \in N_{i}(g)}\left(t_{j}^{i}-t_{i}^{j}\right)-\frac{1}{2} f \eta_{i}(g) \geq \pi_{i}\left(g_{-i}\right) .
$$

In order to obtain a suitable stability criterion for a network between a a large number of firms, let the transfer payments and the linking costs in condition (28) be proportional to the number of firms in the industry. Hence, let us specify $f=n \rho$ and $t_{i}^{j}=n \tau_{i}^{j}$, with $\tau_{i}^{j} \geq 0$, for any $i, j \in N$. Moreover, depict the effective market size by a parameter $A$, where $A=\left(\alpha-\gamma_{0}\right) / \sigma$ and $\sigma$ denotes the minimum market size of footnote 7. Finally, multiply the inequalities in condition (28) by $1 / n$. The limit of the obtained condition is necessary and sufficient for stability of network $g$ and both sides of the inequalities have finite limit values as $n$ grows large.

The following result investigates the stability of an efficient network with respect to a profile $\tau_{i}, i \in\{1,2, \ldots, n\}$, linking cost $\rho$, and market size $A$.

Proposition 5. Suppose a large industry $(n \rightarrow \infty)$. Every transfer-stable network of density $0<D(g)<0.5$ is inefficient.

Proof. Suppose to the contrary a network $g^{*}$, with $0<D\left(g^{*}\right)<0.5$, that is efficient. As shown above, network $g^{*}$ has an inter-linked star architecture that induces a degree partition, $\left\{h_{p}, h_{c}\right\}$. Moreover, the peripheral group, $h_{p}$, comprises a share of $\sqrt{1-D}$ of the total number of firms and for each $i \in h_{p}, i k \in g^{*}$ if and only if $k \in h_{c}$. In the following, we prove that $g^{*}$ is not stable against transfers, when firms compete in prices. (The proof for quantity competition follows in the same way and is therefore omitted.) 
For stability of $g^{*}$, any two $k, l \in h_{c}$ should sustain their link $k l \in g^{*}$,

$$
\lim _{n \rightarrow \infty} 2 \frac{\pi_{k}\left(g^{*}\right)-\pi_{k}\left(g^{*}-k l\right)}{n}=\gamma^{2} \frac{A+1-D}{1-\beta} \geq \rho,
$$

such as any $i \in h_{p}$ and $k \in h_{c}$ should sustain $i k \in g^{*}$,

$$
\lim _{n \rightarrow \infty} \frac{\pi_{i}\left(g^{*}\right)-\pi_{i}\left(g^{*}-i k\right)}{n}+\frac{\pi_{k}\left(g^{*}\right)-\pi_{k}\left(g^{*}-i k\right)}{n}=\gamma^{2} \frac{A+1-D-\frac{1}{2} \sqrt{1-D}}{1-\beta} \geq \rho,
$$

and for any two $i, j \in h_{p}$ with $i j \notin g^{*}$,

$$
\lim _{n \rightarrow \infty} 2 \frac{\pi_{i}\left(g^{*}+i j\right)-\pi_{i}\left(g^{*}\right)}{n}=\gamma^{2} \frac{A-1+D+\sqrt{1-D}}{1-\beta} \leq \rho .
$$

Details on the derivation of the limit values can be found in the supplement to this article published on the RJE website (http://www.rje.org/sup-mat.html). It can be readily seen that inequality (30) implies (29), which is a consequence of the convexity of profits in links. Hence, the cost interval is bounded by (30) and (31).

Moreover, stability of $g^{*}$ requires some profile of transfers such that every $i \in N$ maintains its links. It is natural to focus on transfer profiles, which only depend on the position of a firm in $g^{*}$. Hence, for any $k, l \in h_{c}: \tau_{k}^{l}-\tau_{l}^{k}=0$. Moreover, the net flows from the firms in the center to the peripheral firms are identical across all links. Thus, for any $i \in h_{p}$ and $k \in h_{c}: \tau_{k}^{i}-\tau_{i}^{k}=\tau$. Stability of $g^{*}$ requires that a $\tau \in R$ exists such that any peripheral firm does not delete all its $n(1-\sqrt{1-D})$ links,

$$
\lim _{n \rightarrow \infty} \frac{\pi_{i}\left(g^{*}\right)-\pi_{i}\left(g_{-i}^{*}\right)}{n^{2}(1-\sqrt{1-D})}+\tau=\gamma^{2} \frac{2 A+1-2 D-\sqrt{1-D}}{4(1-\beta)}+\tau \geq \rho .
$$

Furthermore, any firm in the center should not delete all its $n-1$ links,

$$
\lim _{n \rightarrow \infty} \frac{\pi_{k}\left(g^{*}\right)-\pi_{k}\left(g_{-k}^{*}\right)}{n(n-1)}-\lim _{n \rightarrow \infty} \frac{n \sqrt{1-D}}{n-1} \tau=\gamma^{2} \frac{2 A+1+2 D}{4(1-\beta)}-\tau \sqrt{1-D} \geq \rho .
$$

On the one hand, by comparison of (31) and (32) it follows that an appropriate $\tau$ must be positive, which means that the center firms sponsor links to the periphery. In fact,

$$
\tau \geq \gamma^{2} \frac{2 A-5+6 D+5 \sqrt{(1-D)}}{4(1-\beta)} .
$$

On the other hand, $\tau$ is bounded from above by (33). It can be easily verified that for any $A>1$ and $0<D<0.5$, there does not exist any $\tau$ satisfying inequalities (31), (33), and (34) simultaneously, which shows that the inter-linked star architecture is not stable against transfers.

\section{References}

Arrow, K. (1962): "Economic Welfare and the Allocation of Resources for Invention." in The Rate and Direction of Inventive Activity: Economic and Social Factors, ed. by R. Nelson. (NBER) Princeton University Press. 
Bergstrom, T. and Varian, H. "When Are Nash Equilibria Independent of the Distribution of Agents' Characteristics." The Review of Economic Studies, Vol. 52 (1985), pp. $715-718$.

BLOCH, F. "Endogenous Structures of Association in Oligopolies." RAND Journal of Economics, Vol. 26 (1995), pp. 537-556.

Dasgupta, P. And Stiglitz, J. "Industrial Structure and the Nature of Innovative Activity." The Economic Journal, Vol. 90 (1980), pp. 266-293.

D'Aspremont, C. And Jacquemin, A. "Cooperative and Noncooperative R\&D in Duopoly with Spillovers." The American Economic Review, Vol. 78 (1988), pp. 11331137.

Derö̈an, F. And Gannon, F. "Quality-improving alliances in differentiated oligopoly." International Journal of Industrial Organization, Vol. 24 (2006), pp. 629-637.

Duysters, G. And VAnhaverbeke, W. "Strategic Interactions in DRAM and RISC Technology: a Network Approach." Scandinavian Journal of Management, Vol. 12 (1996), pp. 437-461.

Goyal, S. And Joshi, S. "Networks of collaboration in oligopoly." Games and Economic Behavior, Vol. 43 (2003), pp. 57-85.

"Unequal connections." International Journal of Game Theory, Vol. 34 (2006), pp. 319-349.

Goyal, S. And Moraga-GonzÁles, J. "R\&D Networks." RAND Journal of Economics, Vol. 32 (2001), pp. 686-707.

Hagedoorn, J. And Schakenraad, J. "Leading companies and networks of strategic alliances in information technologies." Research Policy, Vol. 21 (1992), pp. 163-190.

Katz, M. "An Analysis of Cooperative Research and Development." RAND Journal of Economics, Vol. 17 (1986), pp. 527-543.

Leahy, D. and Neary, P. "Public Policy Towards R\&D in Oligopolistic Industries." The American Economic Review, Vol. 87 (1997), pp. 642-662.

Powell, W., Koput, K., White, D. And Owen-Smith, J. "Network Dynamics and Field Evolution: The Growth of Interorganizational Collaboration in the Life Science." American Journal of Sociology, Vol. 110 (2005), pp. 1132-1205.

Singh, N. And Vives, X. "Price and Quantity Competition in a Differentiated Duopoly." RAND Journal of Economics, Vol. 15 (1984), pp. 546-554.

SNIJDERs, T. "The degree variance: an index of graph heterogeneity." Social Networks, Vol. 3 (1981), pp. 163-174. 
Wasserman, S. And Faust, K. Social Network Analysis: Methods and Application. Cambridge University Press, 1994.

Westbrock, B. (2008): "Natural Concentration in Industrial Research Collaboration." Discussion Paper no. 08-15, Tjalling C. Koopman Institute, Utrecht University.

YI, S.-S. "Endogenous Formation of Joint Ventures with Efficiency Gains." RAND Journal of Economics, Vol. 29 (1998), pp. 610-631. 